\title{
Lidil
}

Revue de linguistique et de didactique des langues

$31 \mid 2005$

Corpus oraux et diversité des approches

\section{(Dés)alignements en clôture}

Une étude interactionnelle de corpus de français parlé en interaction

\section{Lorenza Mondada et Véronique Traverso}

\section{(2) OpenEdition}

Journals

Édition électronique

URL : http://journals.openedition.org/lidil/128

DOI : $10.4000 /$ lidil. 128

ISSN : 1960-6052

Éditeur

UGA Éditions/Université Grenoble Alpes

Édition imprimée

Date de publication : 1 juin 2005

Pagination : 35-59

ISBN : 2-914176-12-0

ISSN : $1146-6480$

Référence électronique

Lorenza Mondada et Véronique Traverso, " (Dés)alignements en clôture », Lidil [En ligne], 31 | 2005, mis en ligne le 03 octobre 2007, consulté le 01 mai 2019. URL : http://journals.openedition.org/ lidil/128; DOI : 10.4000/lidil.128

Ce document a été généré automatiquement le 1 mai 2019.

(c) Lidil 


\title{
(Dés)alignements en clôture
}

\author{
Une étude interactionnelle de corpus de français parlé en interaction
}

\author{
Lorenza Mondada et Véronique Traverso
}

Corpus de Langue Parlée en Interaction et linguistique interactionnelle

1 L'intérêt croissant pour l'interaction sociale au sein de la communauté scientifique, en sciences du langage mais aussi dans d'autres disciplines comme la sociologie ou l'histoire, a débouché sur deux développements importants :

- d'une part, la reconnaissance des données « naturelles », c'est-à-dire des enregistrements d'activités se déroulant dans leur contexte social de production sans être provoquées par le chercheur, comme objet d'étude de plein droit. Cette évolution a entre autres pour effet l'augmentation de la part, encore largement minoritaire, des données orales interactionnelles dans les grands corpus;

- d'autre part, la découverte des spécificités de ces données et de l'importance théorique de leur prise en compte, qui a conduit au développement de courants de recherche intégrant l'étude de l'organisation interactionnelle dans leurs préoccupations analytiques.

2 Ces courants relèvent, en France comme ailleurs, de différents domaines - de l'analyse du discours (Roulet et alii, 1985, 2001 ; Coulthard, 1992 ; van Dijk, 1997), à la pragmatique des interactions verbales (Kerbrat-Orecchioni 1990, 1992, 1994) à la logique interlocutoire (Trognon, 1999), à l'analyse conversationnelle (Sacks, 1992 ; Sacks, Schegloff, Jefferson, 1974). Inspirée surtout de cette dernière, la linguistique interactionnelle contemporaine (Auer et alii, 1999; Selting \& Couper-Kuhlen, 2002; Ford, Fox \& Thompson, 2002) développe depuis quelques années un corps de connaissances sur l'interaction en tant que pratique sociale et pratique grammaticale, grâce à des analyses qui se sont focalisées à la fois sur l'identification des ressources linguistiques et interactionnelles utilisées par les participants dans leurs échanges et sur la mise en lumière du caractère systématique de leur mobilisation au sein de l'organisation séquentielle de l'interaction. L'étude de la façon méthodique dont les participants constituent l'ordre de leurs conduites a permis de dégager des procédés récurrents, et aujourd'hui, grâce à la variété des corpus étudiés, d'envisager la formulation de généralisations. Elle oriente aussi vers des réflexions sur le caractère (re) configurant de l'interaction pour les ressources linguistiques (Selting \& Couper-Kuhlen, 2000 ; Mondada, 2001, 2005c). 
La démarche d'analyse en linguistique interactionnelle

Les différents courants qui viennent d'être cités partagent un certain arrière-plan commun, tout en accordant une prééminence théorique et analytique à certains aspects de l'interaction plutôt qu'à d'autres, qui se manifeste en retour dans des exigences méthodologiques propres. La démarche de la linguistique interactionnelle, dont sont inspirées les analyses qui suivent, se fonde notamment sur les aspects suivants :

- sur les pratiques des participants avant que sur les formes qu'elles mobilisent : l'objectif premier est moins d'établir des inventaires de formes langagières ou plus largement de ressources interactionnelles que de dégager les modes récurrents de leur mise en œuvre temporelle, séquentielle et collaborative au sein de configurations complexes définies par des positions séquentielles et des agencements de formes;

- sur l'orientation des participants vers les détails qui organisent ces pratiques - détails seen but unnoticed, exploités pour donner sens à ce qui se passe et pour projeter et organiser ce qui y fait suite - et sur l'organisation séquentielle de ces détails ;

- la dimension temporelle de la parole en interaction, qui émerge et se transforme de manière située au fur et à mesure de son déroulement et qui invite à « retemporaliser » non seulement les usages mais aussi la grammaire (Auer et alii, 1999);

- la dimension interactionnelle de cette production, en tant qu'elle se construit de manière collective dans l'ajustement des contributions respectives ;

- la dimension multimodale et non exclusivement verbale de ces pratiques (Mondada, 2005b). Une banque de données de corpus de langue parlée en interaction : CLAPI

Ces caractéristiques de la démarche exigent un traitement technologique spécifique des données. Les données collectées (et numérisées) sont transcrites selon des conventions qui permettent de conserver les caractéristiques fondamentales de l'oral interactif, indispensables pour l'analyse, et qui constituent les détails exploités par les participants eux-mêmes pour organiser leur action. Dans le cadre de la linguistique interactionnelle telle qu'elle vient d'être esquissée ci-dessus, ces exigences s'expriment plus spécifiquement dans l'élaboration de transcriptions d'un degré de granularité fin' en ce qui concerne la séquentialité, d'un système de notation pour les gestes (voir ci-dessous), et de transcriptions alignées avec les enregistrements correspondants qui facilitent la description détaillée des pratiques multimodales grâce à un accès direct de la transcription au son et à la vidéo.

5 Ces exigences sont intégrées dans l'enrichissement progressif de la base CLAPI et ont porté à un ensemble de projets scientifiques qui en exploitent les spécificités au laboratoire ICAR où la base est développée. La base de données est présentée par Balthasar et Bert ici même; nous nous focaliserons ici sur un exemple d'analyse qu'il est possible d'envisager dans cet environnement.

Le phénomène étudié : alignement/désalignement en clôture

6 Dans différents corpus de réunions de travail, nous avons observé que les participants s'orientaient de manière reconnaissable vers la projection de la clôture imminente d'une phase d'activité ou d'un topic (par exemple un point de l'ordre du jour) et qu'ils l'anticipaient de différentes manières, soit en s'alignant (en convergeant vers la clôture projetée), soit en manifestant un « désalignement », par la proposition d'autres trajectoires séquentielles, notamment en prolongeant la phase ou le topic précédent ou en y revenant. 
7 Ce phénomène nous intéresse dans la mesure où il concerne une série de dimensions fondamentales de l'organisation de l'interaction : il repose sur le caractère reconnaissable du découpage de l'activité en phases distinctes et des moyens mis en œuvre pour les délimiter; il concerne les modalités - les méthodes - par lesquelles les participants organisent leur arrivée conjointe à certains points structurants de l'activité et notamment à la fin d'une phase d'activité ; il touche à la possibilité pour les interactants d'exhiber des orientations divergentes quant à la gestion de l'activité. Il nous intéresse aussi parce qu'il est attesté dans une variété de corpus et qu'il semble pourvu de caractéristiques systématiques.

Nous l'abordons ci-dessous en observant successivement l'environnement séquentiel qui le caractérise, puis les ressources (verbales et gestuelles) mobilisées par les participants pour l'organiser. Nous esquisserons ensuite quelques pistes concernant les trajectoires alternatives possibles provoquées par le désalignement.

Ces descriptions nous permettront d'aborder non seulement des questions d'analyse mais de souligner aussi l'importance des modes d'enregistrement et de transcription des données.

Environnement séquentiel

Dans leur article princeps, Schegloff \& Sacks (1973) ont montré l'importance de procédés pour clore une conversation et les problèmes que cette clôture posait aux participants : ceux-ci doivent notamment organiser leur arrivée coordonnée et conjointe à un moment où la conversation n'est plus nourrie en topics et peut donc s'achever : ils le font en initiant une séquence de pré-clôture, suivie de la clôture proprement dite. Ces analyses ont stimulé surtout des études portant sur la fin des interactions ; la clôture de thèmes ou de phases d'activités particulières font recours à des procédés analogues, mais ont plus rarement fait l'objet de recherches. Celles-ci ont néanmoins permis d'identifier des ressources utilisées de façon récurrente par les participants pour effectuer le passage d'un thème ou d'une activité à une autre. Elles ont aussi montré que "réaliser la transition " d'un thème ou d'une phase à un autre constitue un type particulier d'activité conjointe des participants dans l'interaction (Bruxelles, Greco, Mondada, à paraître ; Mondada, 2003 ; Traverso, 1996, 2004, à paraître).

Alignement en clôture

11 Un premier extrait nous permettra de mettre en évidence l'environnement séquentiel d'une clôture de phase d'activité vers laquelle convergent tous les participants.

12 Il s'agit d'une réunion d'équipe dans un service de psychiatrie, où l'on discute successivement le cas de chaque partient :

(1) Corpus Psyc (Mondada) (psyc1/12'50)

\begin{tabular}{|l|l|}
\hline 1 & $(1.6)$ \\
\hline \hline 2 DrL & [voilà $\backslash$ \\
\hline \hline 3 DrD & bo [n euh donc euh on a pas fini d'en \\
\hline \hline 4 & entendre parler de monsieu:r (.) david \\
\hline
\end{tabular}




\begin{tabular}{|l|l|}
\hline 5 DrL & 'ah non ${ }^{\circ}$ \\
\hline \hline 6 DrD & ' $\mathrm{Xx}^{\circ}$ \\
\hline \hline 7 & $(2.4)$ \\
\hline \hline 8 DrD & alo:rs/(.) monsieur gauthier $\backslash$ \\
\hline \hline $9 \mathrm{INT}$ & alors bon moi j'sais pas j'voudrais \\
\hline \hline 10 & interroger l'équipe parce que m- m(oi) sur \\
\hline \hline 11 & le plan euh (0.25) euh moteur/ je le trouve \\
\hline \hline 12 & pas plus mal qu'au début moi \ même plutôt \\
\hline \hline 13 & un petit peu mieux/ \\
\hline
\end{tabular}

13 Après une pause (1) qu'aucun participant ne traite comme une opportunité pour ajouter des informations ou des commentaires sur le cas précédemment traité, deux médecins initient une forme de pré-clôture, le Dr Laurencin par "voilà \» (2) en chevauchement avec le Dr Dumarsais qui produit un commentaire global sur le cas (ce type de maxime, recourant souvent à des expressions figées, relevant du sens commun et visant à susciter l'accord des participants, se trouve souvent dans cette position séquentielle, cf. les «proverbial or aphoristic formulations » de Schegloff and Sacks, 1973, Drew \& Holt, 1998). Le commentaire est suivi par un bref tour qui manifeste un accord (5) et par un autre tour qui, comme lui, est prononcé à voix basse : la diminution du volume de la voix ainsi que la briéveté des tours marquent l'orientation des participants vers le fait que le cas touche à sa fin et ne fera plus l'objet de développements ultérieurs. Suit en effet une longue pause (7), avant que la phase suivante ne soit initiée par le Dr Dumarsais (8), consistant dans l'annonce du patient suivant, projetant son développement topical par l'interne (9sv).

La structure séquentielle à laquelle nous avons affaire est donc la suivante :

15 1. projection de la clôture imminente d'une phase, réalisée par différentes ressources ;

2. alignement des autres participants sur la clôture ainsi initiée ;

3. introduction de la phase suivante ;

4. alignement des participants sur cette nouvelle phase.

Désalignement en clôture

Dans un certain nombre de cas, tous les participants ne convergent pas vers la clôture, ce qui se manifeste par la production d'un enchaînement séquentiel différent que celui qui vient d'être projeté.

17 Voici un cas tiré du même corpus que le précédent : 
(2) Corpus Psyc (Mondada) (psyc3 - 43'54)

\begin{tabular}{|c|c|}
\hline $1 \mathrm{DrD}$ & et pis un neurothymique type \\
\hline 2 & dopamine $\mathrm{p}$ [is c'est bon $\backslash$ \\
\hline $3 \mathrm{DrL}$ & [voilà \\
\hline 4 & $(0.3)$ \\
\hline 5 DrL? & ${ }^{\circ} \operatorname{Xxxxxxxxxx}^{\circ}$ \\
\hline $6 \mathrm{DrD}$ & $x x$ dopamine pouvant entraîner des $(0.5)$ \\
\hline 7 & chutes de la (lignée) sanguine ${ }^{\circ} \mathrm{xx}$ \\
\hline 8 & 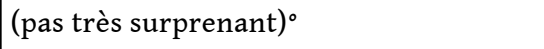 \\
\hline $9 \mathrm{DrL}$ & hhh \\
\hline 10 & $(1.0)$ \\
\hline $11 \mathrm{DrL}$ &.${ }^{\circ \circ} \mathrm{xxxxxxx}^{\circ \circ} \mathrm{hhh}$ \\
\hline 12 & $(0.4)$ \\
\hline 13 DrL & oké hein bo[n \\
\hline $14 \mathrm{DrD}$ & {$[\mathrm{BO}: \mathrm{N} /$ clara/ } \\
\hline 15 & cla[ra spark [a donc \\
\hline $16 \mathrm{INF}$ & [au niveau [au niveau de l'(imovan) \\
\hline 17 & par contre/ (.) $\quad[\mathrm{xxxxx}$ \\
\hline $18 \mathrm{DrL}$ & {$\left[{ }^{\circ}\right.$ ouais $^{\circ}$} \\
\hline 19 DrL & (si tu veux) $\mathrm{xx} \quad[\mathrm{xx}$ \\
\hline $20 \mathrm{INF}$ & [non [supprimer] \\
\hline $21 \mathrm{DrL}$ & [ah bon on] supprime \\
\hline $22 \mathrm{X}$ & un $[$ demi-xxx \\
\hline $23 \mathrm{INF}$ & [parce que (.) elle ouais mais elle \\
\hline
\end{tabular}




\begin{tabular}{|c|c|}
\hline 24 & lui a donné déjà un demi depuis mais elle \\
\hline 25 & dit que : (0.3) ça l'air d'être un rituel/ \\
\hline 26 & il le prend/ elle lui en donnerait deux \\
\hline 27 & ce serait pareil \\
\hline [quoi/ & \\
\hline $28 \mathrm{DrL}$ & {$\left[\right.$ d'ac $^{\circ}$ oké ${ }^{\circ}$} \\
\hline $29 \mathrm{INF}$ & donc elle deman[dait si : (.) ce \& \\
\hline $30 \mathrm{X}$ & {$\left[{ }^{\circ} \mathrm{xx}\right.$ clara $\mathrm{xx}^{\circ \circ}$} \\
\hline $31 \mathrm{INF}$ & \& serait [pas utile de \\
\hline $32 \mathrm{DrL}$ & [de la dopamine (il ; on) \\
\hline 33 & en a $\mathrm{xx}$ vingt-deux globalement \\
\hline 34 SUR & $\mathrm{xxx}$ vingt \\
\hline 35 DrD & voilà \\
\hline 36 SUR & ouais c'est ça \\
\hline 37 & $(0.8)$ \\
\hline $38 \mathrm{DrL}$ & $\mathrm{xxx}$ \\
\hline 39 & $(0.3)$ \\
\hline $40 \mathrm{DrD}$ & alors/ clara sparka/ \\
\hline $41 \mathrm{DrL}$ & ${ }^{\circ} \mathrm{Xx}$ marqué arrêt global de la dopamine ${ }^{\circ}$ \\
\hline 42 DrD & donc y a eu un entretien intéressant \\
\hline 43 & euh avec la famille/ \\
\hline
\end{tabular}

Dans cet extrait on trouve, après la clôture (« oké hein bo[n - [BO:N » 13-14), l'initiation du cas suivant (14-15), chevauchée par l'infirmière (16) qui poursuit la discussion sur les médicaments à administrer au patient précédent (16, cf. 1, 6). L'ouverture du cas suivant est donc posticipée, suspendue par la continuation et la conclusion de la discussion précédente. L'ajout de l'infirmière est formulé par un cadrage thématique (" au niveau de (l'imovan) »16) qui à la fois continue le topic des médicaments à administrer au patient 
(cf. 1) et contraste avec le dernier médicament cité ("par contre» 17). L'infirmière manifeste par là que le cas précédent n'est pas complet, la liste des médicaments n'étant pas épuisée à cet endroit. Les moments de transition révèlent ainsi l'analyse livrée par les participants de la complétude de ce qui précède (Mondada, 2005a).

Durant cet ajout après la clôture, un participant exhibe son orientation vers la phase suivante (30) en reproposant le nom de la patiente à venir; elle ne sera introduite qu'après une nouvelle clôture (35-39) marquée par des accords, des silences, des baisses de la voix, à la ligne 40. De manière intéressante, cette initiation est à nouveau perturbée par un nouvel ajout concernant le cas précédent (41), formulé à voix basse et sans manifester une orientation vers une nouvelle réouverture du cas.

Le désalignement vers une clôture projetée peut se manifester plus ou moins précocement et par diverses ressources langagières. Il peut notamment être introduit par marqueurs d'opposition, voire par des verbes métadiscursifs, comme dans ces deux extraits d'une réunion de recherche entre linguistes :

(3) Corpus Redche (Traverso) (19_041A.28.206)

\begin{tabular}{|c|c|}
\hline 1 & $(2.7)$ \\
\hline 2 Anne & hein \donc i définit/ le cadre \\
\hline 3 & participatif en termes de (.) accès- \\
\hline 4 & accès perceptuel/ (0.8) par rapport auquel \\
\hline 5 & on a un sta- certain statut (1.0) bon $\backslash$ \\
\hline 6 & (0.5). $\mathrm{h}$ alors/(.) voilà les problèmes/ \\
\hline 7 & que [déjà/ moi \\
\hline 8 René & [alors que : Anne c'est i- $\quad$ [c'est idiot \\
\hline 9 Anne & {$[$ (pardon) } \\
\hline 10 & mais par exemple si tu filmes $\backslash(0.4)$ \\
\hline 11 Anne & ouais alors attends j'vais mettre à côté \\
\hline 12 & sur une page/ (un brouillon) tous les \\
\hline 13 & problèmes que ça pose $\backslash$ \\
\hline 14 & (0.9) \\
\hline
\end{tabular}

21 Dans ce premier extrait, Anne prend en charge le passage d'une phase à l'autre de la réunion : on observe la clôture de phase précédente (la définition de la notion de cadre 
participatif) par un énoncé bilan, 2 à 5 (à rapprocher des maximes évoquées ci-dessus), suivi d'une longue pause (5) puis de plusieurs marqueurs remplissant la transition entre les deux phases «bon $\backslash(0.5)$. $\mathrm{h}$ alors/(0.1) », et de l'introduction de la nouvelle phase (les problèmes) en 6 . L'intervention désalignée de René (8) intervient en chevauchement dès que le thème de la nouvelle phase est énoncé. Elle est clairement marquée comme désalignée par le marqueur oppositif "alors que », qui ne vient pas compléter une structure syntaxique entamée et qui n'en ouvre pas (il est posé comme marqueur de prise de tour). Elle est d'autre part signalée comme non préférée par différents indices, tels que l'emploi du terme d'adresse, le petit commentaire "c'est idiot mais» et les autoréparations en début de tour "c'est- c'est idiot ", qui retardent le moment où le point introduit (le cas des films, 10) est énoncé.

(4) Corpus Redche (Traverso) (19_041A.33.42)

\begin{tabular}{|c|c|}
\hline 1 Anne & bon $\backslash$ alors $/(0.2)$ maint'nant (0.4) si le :- \\
\hline 2 & euh é- on va r'garder \\
\hline 3 & juste le format d'réception/ \\
\hline 4 & (.) \\
\hline 5 Léa & mais sauf que/ j'rev-a be j'reviens un \\
\hline 6 & peu en arrière excuse-moi mais sur statut \\
\hline 7 & interlocutif (1.3) statut interlocutif/ \\
\hline 8 & ça réduit encore (.) quand même (0.8) \\
\hline 9 & ça réduit pa'ce que ça ne:- ça ne:: \\
\hline 10 & [prend en compte que l'accès (.) ça ne\& \\
\hline 11 Anne & [ben statut interlocutif/ \\
\hline 12 Léa & \& prend en compte que l'accès \\
\hline 13 Léa & ve- enfin (1.5) auditif et verbal $\backslash$ \\
\hline 14 & (0.5) avec le [mot interlocutif/ \\
\hline 15 Anne & [alors attends \\
\hline 16 & donc/ y a émetteur locuteur hein/ \\
\hline
\end{tabular}

Dans ce second extrait, la clôture de la phase précédente a eu lieu un moment avant, puis quelques échanges se sont déroulés avec une personne extérieure à la réunion qui a fait 
irruption dans la pièce. La phase suivante est introduite de façon très explicite «maint'nant (0.4) si le :- euh é- on va r'garder juste le format d'réception/» (1-3). C'est après une courte pause suivant cette explicitation du thème (4) que Léa produit un tour désaligné (5), introduit là encore par deux marqueurs d'opposition « mais sauf que », par une formulation de ce qui est en train d'être fait (verbe métadiscursif) «je reviens un peu en arrière ». On note comme précédemment l'auto-réparation et une excuse.

De manière générale, la structure séquentielle à laquelle nous avons affaire est la suivante :

1. projection de la clôture imminente d'une phase, voire accomplissement de la préclôture et passage à l'ouverture de la phase suivante ;

2. désalignement par rapport à ce qui est projeté ou initié ;

3. continuation de la phase précédente, voire réouverture.

25 La manifestation du désalignement peut intervenir à des points séquentiels différents, notamment en anticipant plus ou moins sur ce qui est projeté. Ainsi, si A et B sont les deux activités, phases ou thèmes successifs :

Positions séquentielles où un autre participant peut continuer ou reprendre $\mathrm{A}$

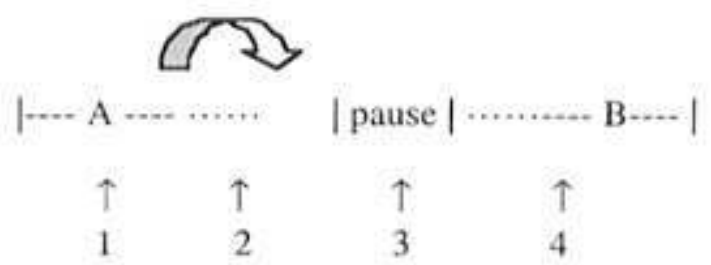

Les extraits cités montrent des positions «tardives» pour la manifestation du désalignement, plutôt vers la position 4 :

- l'un se déroule en chevauchement avec l'introduction de l'activité B. Dans ce cas on observe que la prise de tour désalignée se situe avant que l'introduction du nouveau thème soit tout à fait achevée (extraits 2 et 3 ) ;

- l'autre se déroule une fois cette introduction effectuée (extrait 4).

D'autres positions seront traitées ci-dessous.

Ressources linguistiques mobilisées pour accomplir le désalignement

Ces extraits, choisis parmi une collection de cas analogues, permettent de dégager un certain nombre de ressources utilisées par les participants pour accomplir le désalignement.

- Positionnement séquentiel de la prise du tour désaligné. Le locuteur qui se désaligne peut exploiter des frontières d'unités : il peut intervenir lors de pauses, qu'elles soient situées entre deux activités (en position 3 dans le schéma, comme dans l'extrait 5 infra) ou au point de complétion de la première unité (turn constructional unit, TCU) composant le tour introducteur de B (comme dans l'extrait 4). Il peut aussi introduire son tour en chevauchement: soit lorsque la fin de A est reconnaissablement projetée, en anticipation de la réalisation effective de la clôture (avant l'arrivée à la pause de la position 3); soit avant la complétion de la première unité (TCU) de B. La prise de tour désalignée peut ainsi être qualifiée de précoce ou de tardive selon son positionnement séquentiel.

- Marqueurs de (re)cadrage ou de continuation topicale ou bien marqueurs d'opposition (connecteurs qui ne sont pas intégrés syntaxiquement et sont utilisés comme marqueurs 
de prise de tour, tels que "sauf que» ou "alors que»). Le choix entre ces types de marqueurs est lui aussi lié, entre autres, au positionnement du désalignement.

- Modulations de la voix : le tour désaligné semble être produit de manière à contraster avec le tour sur lequel il enchaîne ou qu'il chevauche. Il se caractérise donc lui aussi selon son positionnement, puisque les caractéristiques prosodiques de la clôture (A) (souvent avec une baisse d'intensité) et celles de l'introduction (B) diffèrent elles aussi.

- Verbes métadiscursifs : plus le désalignement est tardif, plus il est marqué explicitement.

- Dans les positionnements tardifs apparaissent des auto-réparations en début de tour, ainsi que des manifestations du caractère non préféré de l'intervention (termes d'adresse, excuses, commentaires, retardements).

On voit bien qu'une telle analyse repose sur une notation fine des positions séquentielles (attentive notamment à la temporalité des pauses et des chevauchements) ainsi que des détails du segmental : seule leur notation précise peut les rendre disponibles à l'analyse.

Ressources gestuelles

Un autre corpus, enregistré en vidéo, nous permettra d'enrichir la description du phénomène du désalignement par l'observation de la manière dont les participants mobilisent des ressources posturales et gestuelles, outre que matérielles (manipulation d'objets) pour accomplir leurs (dés) alignements. La prise en compte de ces ressources pose d'autres problèmes de transcription, liés en particulier à la notation des gestes, notamment leur délimitation (début/fin) qui implique une discrétisation de mouvements continus.

Les extraits suivants sont tirés d'un enregistrement vidéo d'une réunion où des architectes travaillent sur les plans d'un château qu'ils transforment en hôtel de luxe. Ils sont penchés sur la table, au centre de laquelle sont disposées les planches examinées. La discussion point par point est organisée non seulement verbalement mais aussi par les manipulations des plans.

(5) Corpus Architectes (Projet Mosaic) (11.28.06)

\begin{tabular}{|l|l|}
\hline $1 \mathrm{C}$ & .hhh la seule chose (.) qui serait à modifier \\
\hline \hline 2 & pour l'instant/ .h c'est on sait que y a pas \\
\hline \hline 3 & de (.) de piscine extérieur [e \ \\
\hline \hline $4 \mathrm{M}$ & \\
\hline \hline $5 \mathrm{M}$ & $\mathrm{m}^{*} \mathrm{~m}$ \\
\hline \hline $\mathrm{C}$ & $* .$. \\
\hline \hline 6 & $(0.8)^{*}(1.3)^{*}$ \\
\hline \hline $\mathrm{C}$ & $\ldots . . .{ }^{*}$ met le plan de côté* \\
\hline \hline $7 \mathrm{M}$ & pas extérieure/ *donc y en * \\
\hline
\end{tabular}




\begin{tabular}{|c|c|}
\hline & *...............* \\
\hline 8 & *aura une :/ à l'inté[rieur eh/* \\
\hline & *repose le plan au milieu de la table* \\
\hline $9 \mathrm{C}$ & [à l'intérie*ur \\
\hline $10 \mathrm{M}$ & à l'inté [rieur de la: \\
\hline $11 \mathrm{C}$ & [c'est-à-dire que : iz ${ }^{\wedge}$ estiment \\
\hline 12 & qu'en Bourgogne y a pas de : $(d ; 1) e$ \\
\hline 13 & clima:t euh pour justifier une piscine \\
\hline
\end{tabular}

Dans ce premier extrait, Charles termine son tour d'une manière rendue reconnaissable par sa complétude syntaxique et une intonation conclusive; il est accueilli par des acquiescements minimaux de Marie (4-5) ; pendant la pause qui suit, il met de côté le plan dont il était question (6). Jusqu'ici personne ne continue à alimenter le topic de la piscine - le plan permettant de produire des descriptions indexicales n'est d'ailleurs plus disponible. Néanmoins, après la pause, Marie produit une question se présentant comme un ajout après la complétion du tour précédent, recyclant un fragment de ce tour dans sa forme négative et enchaînant par sa conséquence positive. Immédiatement, après le premier TCU de Marie, Charles remet le plan au milieu de la table et s'aligne donc avec le désalignement de cette dernière, gestuellement avant même que verbalement (11).

Tout de suite après, Charles clôt la question de la piscine en remettant le plan de côté (7) :

(6) Corpus Architectes (Projet Mosaic) (11.28.43) (cont. extr.5)

\begin{tabular}{|c|c|}
\hline $1 \mathrm{C}$ & une construction penda:nt. h toute cette \\
\hline 2 & période-là donc on on on RE:part sur un AU:tre \\
\hline 3 & concept qui est le concept du: ${ }^{\circ} \mathrm{comment}$ ils \\
\hline 4 & appellent ça/euh $\left[\mathrm{h}^{\circ}\right]$ \\
\hline $5 \mathrm{~L}$ & [tsk] euh (ou) une p'tite \\
\hline 6 & $\mathrm{sa}^{\wedge}$ lle de j'sais plus \\
\hline & $\wedge \ldots . . .>$ \\
\hline 7 & ${ }^{*}$ quoi/ (.) on^ ${ }^{\wedge}$ va trou*ver \\
\hline & $\rightarrow \wedge$ feuillette le fax $\rightarrow$ \\
\hline
\end{tabular}




\begin{tabular}{|c|c|}
\hline c & *...................... met le plan de côté $\longrightarrow>$ \\
\hline 8 & $(1.0)$ \\
\hline $9 \mathrm{C}$ & xx *c'est jacuzzi ::s/ [(.) s*au : na :/* \\
\hline & $->{ }^{*},{ }_{, \prime,}, *$ \\
\hline $10 \mathrm{M}$ & [ah oui \\
\hline $11 \mathrm{C}$ & {$[\mathrm{xx}(\mathrm{ehm})$} \\
\hline $12 \mathrm{M}$ & [mais avec un bassin quand même/^ \\
\hline 1 & $\rightarrow \wedge$ \\
\hline $13 \mathrm{C}$ & avec un bass [in $\backslash$ \\
\hline $14 \mathrm{~L}$ & {$[<$ oui mais à } \\
\hline 15 & l'inté $^{\wedge}$ rieur^ $^{\wedge}(($ rapide $))>. h$ \\
\hline & ^.......^^pointe v.plan précédent $\longrightarrow>$ \\
\hline 16 & $\mathrm{y}$ avait une autre chose aussi à \\
\hline 17 & expli^quer c’était l’idée \\
\hline & $->\wedge$ \\
\hline 18 & des :: ${ }^{*}$ des parkings/m (ais) en fait euh*/ \\
\hline $\mathrm{c}$ & *remet le plan au milieu ---_* \\
\hline $19 \mathrm{C}$ & voilà $\backslash$ mais ça finalement pour l'instant \\
\hline 20 & c'est $u[n$ acquis euh ::/ \\
\hline $21 \mathrm{~L}$ & [c'est accepté \} $\\
$\hline
\end{tabular}

Cette fois, Charles met le plan de côté avant la fin de son tour en forme de liste, anticipant ainsi de manière reconnaissable la clôture de ce topic. Marie acquiesce (10) tout en ajoutant immédiatement une nouvelle question (12) qui se présente sous la forme d'une continuation de l'énoncé précédent de Charles, en lui accolant un syntagme prépositionnel qui est aussi une rectification de la liste. Cet élément est confirmé par Charles et par Luc; ce dernier énonce ce premier TCU de manière rapide (14) pour y ajouter un autre (15) qui constitue l'ajout d'un complément par rapport au dernier topic 
( $\mathrm{y}$ avait une autre chose aussi à expliquer»16-17) et qui en pointant vers le plan précédent le rend pertinent: Charles remet alors le plan sur la table.

Alors que dans le premier cas, le geste de Charles était placé durant la pause entre une phase et l'autre de la discussion, dans le second il anticipe la fin du tour final ; mais dans les deux cas une question de Marie suspend la suite et provoque le retour du plan précédent au milieu de l'espace de travail. Ce retour peut d'ailleurs se faire relativement tardivement et par des moyens verbaux et gestuels explicites, comme dans cet extrait :

(7) Corpus Architectes (Projet Mosaic) (11.34.06)

\begin{tabular}{|c|c|}
\hline $1 \mathrm{C}$ & voilà/ ce qu'on voi:t/ c'est qu'on peut quand \\
\hline 2 & même se balade:r/[et on garde l'histoire du \& \\
\hline $3 \mathrm{M}$ & [ouais \\
\hline 4 & \&chemin de ronde un pe[tit peu:/ \\
\hline $5 \mathrm{M}$ & [d'accord \\
\hline $6 \mathrm{C}$ & euh. hh on a cet *espa*ce là :/ \\
\hline & *....*met plan de côté 䜿 \\
\hline 7 & $(2.0)^{*}$ \\
\hline & $->*$ \\
\hline $8 \mathrm{C}$ &.$h$ ça c'était un p'+tit p[eu \\
\hline $9 \mathrm{M}$ & {$[\mathrm{ah}$ ou +} \\
\hline & $+\ldots \ldots \ldots \ldots+\cdots+$ \\
\hline 10 & $+\mathrm{i}$ donc + attends + \\
\hline & +1 main -+2 mains sur le plan préc. + \\
\hline 11 & (.) donc ça/ + ce- cette + façade a été \\
\hline & + bouge le pl. + \\
\hline 12 & complètement re[maniée \\
\hline $13 \mathrm{~L}$ & [voilà \ \\
\hline
\end{tabular}

41 Charles termine sa description et met le plan de côté avant la fin de son tour (6), dans un geste qui se prolonge pendant la pause (7). Il produit ensuite un bilan rétrospectif de ce 
qui a été dit, orienté après coup sur le topic déjà clos - exhibé comme tel par la forme passée du verbe (8). Toutefois à ce moment-là Marie revient sur le plan, à la fois par le marqueur discursif « attends » (10) et en touchant le plan, d'abord avec une main, ensuite des deux mains. Elle tire le plan vers elle durant sa question.

De nouvelles positions séquentielles pour le désalignement apparaissent dans ces extraits.

Positions des désalignements du locuteur suivant

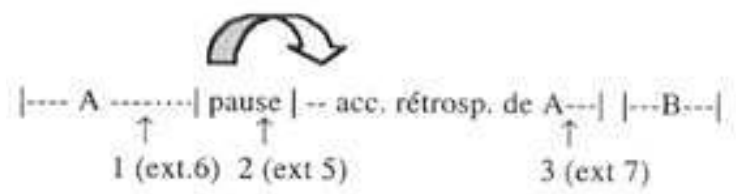

1. la première position est située durant la projection de la fin du tour qui est aussi la fin d'une phase d'activité ou d'un topic ;

2. la seconde se place durant la pause après la clôture ;

3. la troisième se place après la clôture rétrospectivement commentée par le locuteur qui l'a accomplie, traitant la phase précédente comme complétée.

Aux ressources langagières dégagées ci-dessus (2.2) viennent s'ajouter différentes ressources gestuelles liées à la manipulation d'objets, telles que toucher ou déplacer un plan, tracer une ligne, déchirer un calque, prendre un document en main.

Trajectoires séquentielles possibles

Ces analyses ont permis de dégager les caractéristiques de la réalisation des désalignements à partir de leur initiation, précoce ou tardive. Différentes pistes d'investigation s'ouvrent à partir de là, concernant en particulier la trajectoire que va suivre ce qui a été ainsi introduit.

L'analyse de cette trajectoire conduit à s'arrêter en premier lieu à l'enchaînement immédiatement produit après le tour désaligné. Dans les extraits de la réunion de recherche (3 et 4 ) semble se dessiner une tentative de rejet de l'expansion ouverte par le désalignement, alors que dans les deux autres corpus (en particulier dans le corpus Architectes) il y a plutôt alignement immédiat sur le désaligné. Dans un second temps, il convient d'étudier la suite de la trajectoire : elle peut constituer un bref échange ponctuel (visant par exemple à compléter, préciser, apporter ou demander des explications supplémentaires, extraits $2,5,6,7)$ suivi d'une reprise de ce qui était en train d'être effectué, mais dans bien des cas c'est un long développement qui est ainsi ouvert (extraits $3,4)$.

L'étude de ce développement pose de façon centrale la question du lien entre le phénomène de l'alignement/désalignement et la manifestation d'accords/désaccords. En effet, si un désalignement manifeste bien un désaccord sur la structuration de l'activité en cours (un « refus » de clore une phase pour passer à la suivante), cela ne signifie pas nécessairement qu'il exprime un désaccord au niveau des contenus échangés ou des positions argumentatives des participants.

47 Dans certains des extraits étudiés, le tour désaligné introduit un élément manifestant un désaccord sur les contenus: par exemple, dans l'extrait 4, le tour désaligné de Léa conteste le choix du terme «statut interlocutif » : 
(8) Suite de l'extrait (4)

\begin{tabular}{|c|c|}
\hline 4 Léa : & mais sauf que/j'rev- a be j'reviens un \\
\hline 5 & peu en arrière excuse-moi mais sur statut \\
\hline 6 & interlocutif (1.3) statut interlocutif/ \\
\hline 7 & ça réduit encore (.) quand même (0.8) \\
\hline 8 & ça réduit pa'ce que ça ne :- ça ne :: \\
\hline 9 & [prend en compte que l'accès (.) ça ne \& \\
\hline 10 Anne & [ben statut interlocutif/ \\
\hline 11 Léa & \& prend en compte que l'accès \\
\hline 12 & ve- enfin (1.5) auditif et verbal $\backslash(0.5)$ \\
\hline 13 & avec le [mot interlocutif/ \\
\hline 14 Anne & [alors attends \\
\hline 15 & donc/y a émetteur \\
\hline 16 & locu[teur hein/ \\
\hline 17 Inès & {$[<(($ très bas $))$ interlocution oui > } \\
\hline 18 Léa : & on a viré euh : tout c'qui \\
\hline 19 & était [visuel/et : participation \\
\hline 29 Sara & [tout c'qui est visuel \\
\hline 21 Léa & [euh :: non verbale \\
\hline 22 Anne & [alors attends pourquoi :/ \\
\hline
\end{tabular}

Le tour commencé à la ligne 4 se développe avec une mise en place du thème (« sur statut interlocutif ») suivie d'une pause, puis, dans une construction disloquée, de l'explicitation du caractère inadéquat du choix terminologique ("ça réduit encore ", "ça réduit », "ça ne prend en compte que... »). Dans la suite de l'extrait, on voit d'une part que Anne conteste ce qui est en train d'être dit (14), puis cherche à reprendre le tour (15), et d'autre part que les autres participants interviennent dans l'échange en prenant position autour du point de discussion $(17,20)$. 
Dans d'autres cas, le désalignement introduit au contraire une contribution venant renforcer un accord, comme dans l'extrait suivant, tiré de la réunion d'architectes :

(9) Corpus Architectes (Projet Mosaic) (12.06.36)

\begin{tabular}{|c|c|}
\hline 1C: & *vous vous êtes sûrs* qu'il faut garder \\
\hline & 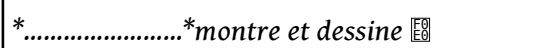 \\
\hline 2 & la double circulation \#vous/ --* \\
\hline & $\rightarrow$ la double circulation -* \\
\hline im & $\#$ \#im1 \\
\hline 3 & $(0.6)$ \\
\hline $4 \mathrm{~L}:$ & oui : $\mathrm{h}$ \\
\hline 5 & $(1.7)$ \\
\hline $6 \mathrm{M}:$ & .h ouais j'trouve ça :: euh \\
\hline $7 \mathrm{C}:$ & vous êtes sûrs/ \\
\hline $8 \mathrm{~L}:$ & $\mathrm{mm}=$ \\
\hline $9 \mathrm{M}:$ & = ouais \\
\hline $10 \mathrm{C}:$ & oui *en plus euh* ça permet \#d'aller aux \\
\hline & *...........*pointe vers zone 1 -> \\
\hline im & \#im2 \\
\hline 11 & sanitai*res là/ \\
\hline & $\rightarrow *$ \\
\hline $\begin{array}{ll}12 \mathrm{M} \\
: & \end{array}$ & ouais \\
\hline $13 \mathrm{C}:$ & bon $\backslash(2.1)$ et le [bar (.) euh :: euh : \\
\hline $14 \mathrm{M}$ : & [ouais \\
\hline 15 & $(4.0)$ \\
\hline $16 \mathrm{C}:$ & le bar on *le met [tou*jours*\# \\
\hline
\end{tabular}




\begin{tabular}{|c|c|}
\hline & $* \ldots \ldots . . . . . *$ pointe vers zone $2^{*}$ \\
\hline im & \#im3 \\
\hline $17 \mathrm{~L}:$ & [c'est pas \\
\hline 18 & *agréa^ ble en* plus \\
\hline & $\wedge_{\ldots . . . .-->}$ \\
\hline c & *,,,",,',",,", \\
\hline 19 & 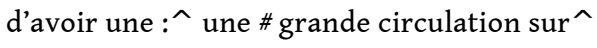 \\
\hline & $\rightarrow \wedge$ montre la double circ. $s / p l-\wedge$ \\
\hline im & \#im4 \\
\hline 20 & ^un grand espace comme ça \\
\hline & ^dessine dans l'espace -> \\
\hline 21 & très en longueur : $\backslash$ \\
\hline $22 \mathrm{C}:$ & ouais $=$ \\
\hline $23 \mathrm{~L}:$ & $=$ une grande circulation qui distribue à \\
\hline 24 & chaque fois euh $::(.)^{\wedge}$ \\
\hline & $\rightarrow \wedge$ \\
\hline $25 \mathrm{C}:$ & oui \en enfilade \\
\hline $26 \mathrm{~L}:$ & (c') pas tr[ès :: là y a\& \\
\hline $27 \mathrm{C}:$ & [oui (c'est moche) \\
\hline $28 \mathrm{~L}$ & $\wedge$ \& une espèce de liberté \\
\hline & ${ }^{\wedge}$ geste sinueux au-dessus du pl $\rightarrow>$ \\
\hline 29 & les gens peuvent ${ }^{\wedge}$ circuler :: \\
\hline & $\rightarrow \wedge$ \\
\hline 30 & [autour des noyaux : fonctionnels/ \\
\hline $31 \mathrm{C}:$ & [oui oui c'est vrai \\
\hline
\end{tabular}




\begin{tabular}{|l|l|}
\hline 32 C : & *c'est vrai c'est vrai c'est vrai* \\
\hline \hline & *dessine s/calque une ligne sinueuse* \\
\hline
\end{tabular}

Dans cet extrait, le tour désaligné intervient dans la position 3, après l'introduction du thème de la phase suivante (13-14) réalisée verbalement «et le bar » et gestuellement, puisque Charles tend le bras vers la zone du calque concernée par l'emplacement du bar (16). Le tour désaligné de L (17) intervient en chevauchement avant que le nouveau thème ne soit totalement inscrit. Il est présenté comme apportant un argument supplémentaire en faveur de l'accord construit conjointement par les trois participants sur le thème précédent ("garder la double circulation », 1-2). Le geste désaligné (image 4, 1.19) revient au même endroit que le geste antérieur de Charles (image 1 et image 2, 1. 2 et 1.10), alors que celui-ci était passé à une autre zone du plan (le bar, image 3, l. 16).

Extrait
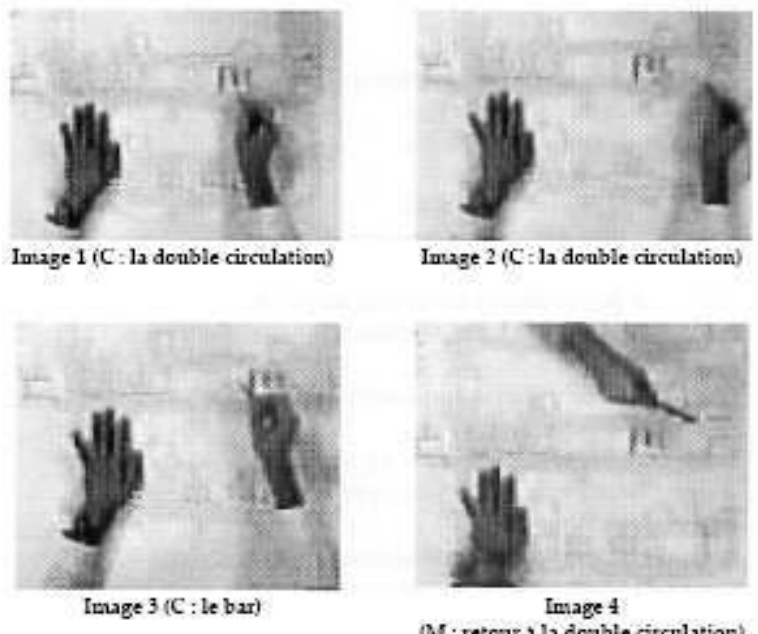

Cet extrait illustre bien le phenomène d'alignement innnédiat sur le désaligné : dès que Louis prend la parole (17-19) et tend son bras vers la zone de la double circulation, Charles recule, puis il contribue à l'expansion introduite : régulation (22), soufflage de mot (25, où Charles prononce le mot « enfilade » correspondant au geste fait dans l'espace par Louis), évaluation en écho (26 L « c'est pas très ::», 27 C « oui c'est moche ») et enfin « inscription» par Charles sur le calque (32) de ce que Louis vient de dessiner dans l'espace avec un geste sinueux (« une espèce de liberté » 28 ).

Le désalignement en clôture est un phénomène particulièrement intéressant pour aborder la question de la relation entre les phénomènes de co-construction de l'interaction sur le plan séquentiel et sur le plan des contenus, qui rejoint par bien des points les débats en cours autour de la notion de négociation (Traverso, 2005). L'étude qui en est proposée ici montre l'importance d'en effectuer une analyse s'appuyant aussi finement que possible sur les détails accessibles aux participants et qu'ils mobilisent, qui seule permet d'établir, dans toute sa complexité, la variété des manifestations du phénomène.

Une autre piste de recherche prometteuse concerne le lien entre le positionnement et la forme du phénomène d'une part et le type d'interaction dans lequel il se produit d'autre 
part. La recherche présentée ici s'est focalisée sur les réunions, et il serait sans aucun doute intéressant de travailler sur des types d'interactions tout à fait différents, par exemple des conversations ordinaires. Mais il est aussi tout à fait important de souligner à la fois la récurrence et la variété des ressources en jeu dans le type « réunion »: selon qu'il s'agit d'une réunion de conception, d'une réunion de recherche ou d'une réunion d'équipe dans un service médical, le phénomème est à la fois analogue et différent. Des études dans ce sens permettront d'affiner la réflexion sur les types d'activités au sein des types d'interactions. Dans la réunion d'architectes par exemple, différentes activités clairement distinctes s'enchaînent - lecture collective d'un document, présentation/ explication du projet, élaboration collective de solutions, (voir Bruxelles, Greco \& Mondada, à paraître ; Détienne \& Traverso, à paraître) - mobilisant différentes ressources matérielles (document lu, pris ou reposé, traçage sur un claque, plan déplacé, etc.) pour leur structuration et donc pour l'accomplissement des clôtures, des alignements ou des désalignements.

Conclusion : vers une technologie de corpus

$\mathrm{Au}$ terme de ce texte, on peut s'interroger sur une linguistique interactionnelle outillée c'est-à-dire dotée de moyens informatiques propres à une technologie pour le traitement des corpus de parole en interaction - qui permettrait d'effectuer ce type d'analyse sur des corpus quantitativement plus importants.

Dans cette perspective, et en visant le développement de moteurs de recherche et d'outils de requête (semi) automatiques, on peut expliciter les entités interrogeables dans les corpus de transcriptions: des formes linguistiques, des notations du multimodal, des caractéristiques de l'oral interactif transcrites par convention (par exemple les chevauchements, les pauses), des annotations de catégories fonctionnelles (clôtures par exemple). La possibilité d'effectuer de telles requêtes repose sur l'établissement de transcriptions à la fois rigoureuses, cohérentes et informatiquement structurées.

C'est à partir de telles prémisses qu'il sera possible d'avancer vers l'objectif de réaliser des requêtes sur les " configurations complexes », c'est-à-dire de faire des recherches sur des conglomérats de phénomènes, produits par le même participant ou des participants différents, qui se situent par rapport à d'autres phénomènes notés par convention (par exemple un tour de parole, un TCU), et qui sont agencés les uns par rapport aux autres dans une certaine organisation temporelle et séquentielle.

Les clôtures et désalignements sont de bons candidats pour travailler dans ce sens (série de «formes », produites par plusieurs participants, dans une certaine séquentialité). De tels phénomènes permettent d'une part de tester la faisabilité de requêtes complexes. Ils permettent d'autre part de préciser l'articulation entre analyse qualitative et analyse quantitative, la première n'étant pas seulement le préalable indispensable de la seconde mais aussi, en définitive, la démarche heuristique qui permet de prendre en compte et de dépasser les limites des langages de requêtes. 


\section{BIBLIOGRAPHIE}

AUER, P., COUPER-KUHLEN, E. et MÜLLER, F. (1999) : Language in Time. The Rhythm and Tempo of Spoken Interaction. Oxford : Oxford University Press.

BRUXELLES, S., GRECO, L., et MONDADA, L. (à paraître) : Procédés interactionnels pour l'accomplissement des transitions au sein des activités de co-conception, in Détienne, F. et Traverso, V. (dir). : Méthodologies d'analyse de situations coopératives de conception. Nancy, PUN. COUlTHARD, M. (1992) : Advances in Spoken Discourse Analysis, London, New York, Routledge. DÉTIENNE, F. et TRAVERSO, V. (dir). (à paraître) : Méthodologies d'analyse de situations coopératives de conception. Nancy, PUN.

DIJK, T.A. van (ed.) (1997) : Discourse as Social Interaction. Discourse Studies 2. A Multidisciplinary Introduction. London : Sage.

DREW, P. et HOLT, E. (1998) : Figures of speech: Figurative expressions and the management of topic transition in conversation. Language in Society, 27, 495-522.

FORD, C.E., FOX, B.A. et THOMPSON, S.A. (eds.). (2002) : The Language of Turn and Sequence. Oxford, Oxford Studies in Sociolinguistics.

KERBRAT-ORECCHIONI, C. $(1990,1992,1994)$ : Les interactions verbales, tomes 1, 2, 3. Paris, Colin. MONDADA, L. (2001) : Pour une linguistique interactionnelle, Marges Linguistiques, $\mathrm{n}^{\circ} 1$, mai 2001. $<\mathrm{http}$ ://www.marges-linguistiques.com> (republié dans M. Santacroce, éd., Faits de langue, faits de discours, Paris, L'Harmattan, vol. 2, 95-136).

MONDADA, L. (2003) : Parler topical et organisation séquentielle : l'apport de l'analyse conversationnelle, Verbum, 25, 2, 193-219.

MONDADA, L. (2004) : Temporalité, séquentialité et multimodalité au fondement de l'organisation de l'interaction : le pointage comme pratique de prise du tour, Cahiers de linguistique française, 26, 169-192.

MONDADA, L. (2005a) : L'ordre interactionnel et l'ordre institutionnel comme accomplissements pratiques des membres dans le temps, Médias \& Culture, 2.

MONDADA, L. (2005b) : L'exploitation située de ressources langagières et multimodales dans la conception collective d'une exposition, in J.-P. Bronckart, L. Fillietaz, éds. L'analyse des actions et des discours en situation de travail. Concepts, méthodes et applications, Louvain, Peeters.

MONDADA, L. (2005c) : L'analyse de corpus dans la perspective de la linguistique interactionnelle : des analyses de cas singuliers aux analyses de collections. In A. Condamine (éd.), Sémantique et corpus, Paris, Hermès.

ROULET, E., AUCHLIN, A., MÆSCHLER, J., SCHELLING M., RUBATTEL, C. (1985) : L'articulation du discours en français contemporain. Berne, Francfort-s. Main, New York, Paris, Peter Lang.

ROULET, E., FILLETTAZ, L, GROBET, A. (2001) : Un modèle et un instrument d'analyse de l'organisation du discours, Bern, Lang.

SACKS, H. (1992) : Lectures on Conversation [1964-72] (2 vols.). Oxford : Basil Blackwell. 
SACKS, H., SCHEGLOFF, E.A. et JEFFERSON, G. (1974) : A simplest systematics for the organization of turn-taking for conversation, Language, 50, 696-735.

SCHEGLOFF, E. et SACKS, H. (1973) : Opening up closings, Semiotica VIII (4), 289-327.

SELTING, M. et COUPER-KUHLEN, E. (2000) : Argumente für die Entwicklung einer « interaktionalen Linguistik ». Gesprächsforschung. Online-Zeitschrift zur verbalen Interaktion, 1, 76-95.

SELTING, M. et COUPER-KUHLEN, E. (eds.). (2002) : Studies in Interactional Linguistics. Amsterdam : Benjamins.

TRAVERSO, V. (1996) : La conversation familière. Lyon, PUL.

TRAVERSO, V. (2004) : Interlocutive « crowding » and « splitting » in polylogues: the case of a researchers'meeting, Journal of Pragmatics, 36, 53-74.

TRAVERSO, V. (2005) : Cristallisation des désaccords et mise en place de négociations dans l'interaction : des variations situationnelles. in M. Grosjean et L. Mondada (dir). Des négociations au travail, Lyon, PUL/ARCI.

TRAVERSO, V. (à paraitre) : Quelques formats intégrant la répétition comme ressource pour le développement thématique dans la conversation ordinaire, in R. Galatolo et A. Fasulo (dir), Special Issue on Conversation Analysis. Rivista di psicolinguistica applicata.

TROGNON, A (1999) : Éléments d'analyse interlocutoire, in M. Gilly, J.-P. Roux et A. Trognon (dir), Apprendre dans l'interaction, Nancy, PUN/PUP, 69-95.

\section{ANNEXES}

Conventions de transcription

[ chevauchements

/ \intonation montante / descendante ।

(2.1) pauses en secondes

(.) micro-pause

exTRA segment accentué

xxx segment inaudible

((rire)) phénomènes non transcrits

: allongement vocalique

< > délimitation des phénomènes entre (( ))

par- troncation

\& continuation du tour de parole

= enchaînement rapide

$\wedge$ liaison

'bon' murmuré

.h aspiration 
h expiration

(il va) transcription incertaine

Notation des gestes (version LM 2.0.4, Mondada, 2004)

* * indication du début/de la fin

^ d'un geste d'un participant

+ + décrit à la ligne suivante

(un symbole par participant)

Si à la ligne suivante ce n'est pas le geste du locuteur mais celui d'un co-participant qui est décrit, alors son initiale figure au début de la ligne en minuscule. S'il s'agit du locuteur en train de parler, il n'y a pas d'initiale.

.... amorce du geste

,", fin/retrait du geste

->> continuation du geste aux lignes suivantes

jusqu'à la flèche suivie de la borne droite

->> continuation du geste

jusqu'au-delà de la fin de l'extrait

>> geste ayant commencé avant le début de l'extrait

\section{NOTES}

1. Différents critères et principes pour la transcription ont été définis dans le cadre du travail autour de la base CLAPI, voir :

$<$ http://icar.univ-lyon2.fr/projets/nomex-clapi/index.html>

2. Les modes de citation des corpus sont ceux établis dans les conventions ICOR (cf. la page internet citée dans la note précédente). Nous donnons une version brève et simplifiée de cette convention à la fin de ce texte.

\section{AUTEURS}

LORENZA MONDADA

Université Lyon 2-ICAR

VÉRONIQUE TRAVERSO

CNRS-ICAR 